\title{
La PME Et L'entrepreneuriat Font-Ils L'objet De Recherche Au Maroc? Une Étude Bibliographique
}

\author{
Omar Taouab \\ Khalifa Ahsina \\ Hamid Ait lemqueddem
}

Enseignant chercheur à l'Ecole Nationale de Commerce et de Gestion,

Kénitra, Maroc

doi: 10.19044/esj.2016.v12n1p242 URL:http://dx.doi.org/10.19044/esj.2016.v12n1p242

\begin{abstract}
The evaluation of the research in SMES and entrepreneurship has been the subject of several research works. These works have been made particularly in the anglo-saxon countries, Busenitz et al (2003), Meyer and al, (2014), Lasch and Yami (2008) in France and Sánchez and Gutiérrez (2011) in Spain. By contrast, no analysis of scientific journals in the field of entrepreneurship has yet been made in Morocco, from where, the desirability of this work, if only by a comparison of the results with the work already cited. This article has thus, for the purpose of presenting the results of a bibliographic study of 237 articles of the three major journals of Moroccan management on 2005-2013. The results suggest that the research in entrepreneurship is still low with a rate of $12 \%$ of the whole research in management. That the review of the Moroccan trade and management made more than $62 \%$ of this production volume, and the qualitative methodology is the most used.
\end{abstract}

Keywords: SMES, entrepreneurship, evaluation of the research, Morocco

\section{Résumé}

L'évaluation de la recherche en PME et entrepreneuriat a fait l'objet de plusieurs travaux de recherche. Ces travaux ont été réalisés notamment dans les pays anglo-saxons, Busenitz et al (2003), Meyer et al, (2014), Lasch et Yami (2008) en France et Sánchez et Gutiérrez (2011) en Espagne. Par contre, aucune analyse des revues scientifiques dans le domaine de l'entrepreneuriat n'a encore été faite au Maroc, d'où, l'opportunité de ce travail, ne serait-ce que par une comparaison des résultats avec les travaux déjà cités. Cet article a donc, pour but de présenter les résultats d'une étude bibliographique de 237 articles des trois principales revues de gestion 
marocaines sur la période 2005-2013. Les résultats dégagés laissent entendre que la recherche en entrepreneuriat est encore faible avec un taux de $12 \%$ de l'ensemble de la recherche en gestion. Que la revue marocaine du commerce et de gestion fait plus de $62 \%$ du volume de cette production, et la méthodologie qualitative est la plus utilisée.

Mots clés : PME, entrepreneuriat, évaluation de la recherche, Maroc

\section{Introduction}

Le champ de la recherche en entrepreneuriat a connu une croissance exponentielle depuis plus d'un demi-siècle (Fayolle, 2000). En effet, d'à peine une poignée de revues il y a 30 ans on a dépassé la barre de 100 .

En effet, pour Aldrich et Baker (1997), la recherche en PME et entrepreneuriat a commencé à attirer l'intérêt des chercheurs, mais en tant que domaine de recherche elle n'a réalisé que des progrès limités en tant que discipline scientifique normale.

De même, Harrison et Leitch (1996) ont constaté que les recherches sur l'entrepreneuriat publiées dans les revues de gestion de 1987 à 1993 représentent un très petit pourcentage de toute la recherche sur ce domaine, et que la grande majorité de ces recherches est publiée dans des revues dédiées à l'entrepreneuriat et la petite entreprise.

Au Maroc, la création d'entreprise reçoit de plus en plus d'attention de la part des décideurs et des chercheurs marocains.

En effet, elle est devenue depuis plus d'une décennie une stratégie privilégiée des pouvoirs publics (O, Taouab, 2014).

Or, la formation et la recherche en gestion ont débuté tardivement, dans les années $\subseteq$ L'évaluation de la recherche en PME et entrepreneuriat a fait l'objet de plusieurs travaux de recherche. Ces travaux ont été réalisés notamment dans les pays anglo-saxons, Busenitz et al (2003), Meyer et al, (2014), Lasch et Yami (2008) en France et Sánchez et Gutiérrez (2011) en Espagne.

Par contre, aucune analyse des revues scientifiques dans le domaine de l'entrepreneuriat n'a encore été faite au Maroc, d'où, l'opportunité de ce travail, ne serait-ce que par une comparaison des résultats avec les travaux déjà cités.

Donc, la question principale à laquelle ce travail va essayer d'y apporter une réponse est la suivante : "la PME et l'entrepreneuriat font t'ils l'objet de recherche au Maroc? ».

De cette question principale découle les questions suivantes :

- Quelles sont les revues où on publie le plus d'articles dans ce domaine ?

- Quels sont les chercheurs les plus productifs?

- Quels sont les thèmes les plus étudiés?

Et enfin quelles sont les méthodologies les plus utilisées? 
Pour répondre à cette problématique, notre article sera structuré de la manière suivante : il commence par une revue succincte de la littérature portant sur l'évaluation de la recherche en entrepreneuriat (section 2). Elle se poursuit par la description de la méthodologie (Section 3). Les résultats obtenus sont exposés ensuite (section 4). Et pour conclure on discutera des principaux résultats.

\section{Revue de la littérature}

$\mathrm{Vu}$ la jenesse de la discipline au niveau marocain on se contentera de la revue des principales études qui ont été fait au niveau des pays développés.

Tableau 1: Les recherches en Entrepreneuriat

\begin{tabular}{|c|c|c|c|c|}
\hline Auteurs & Échantillon & Période & $\begin{array}{l}\text { Méthodol } \\
\text { ogie }\end{array}$ & Résultats \\
\hline Busenitz et al & $\begin{array}{l}97 \text { articles } \\
\text { dans } 7 \\
\text { revues } \\
\text { majeures en } \\
\text { managemen } \\
\text { t }\end{array}$ & $\begin{array}{c}\text { de } 1985 \text { à } \\
1999\end{array}$ & \begin{tabular}{|l} 
Utilisatio \\
n de mots \\
clés \\
comme \\
l'entrepre \\
neuriat \\
ou un \\
mot \\
similaire \\
\end{tabular} & $\begin{array}{l}\text { - les articles publiés dans ce } \\
\text { champ de recherche représentent } \\
\text { moins de } 2 \% \text { de tous les articles } \\
\text { publiés en management, } \\
\text { - ce taux a tendance à augmenter, } \\
\text { - et enfin, les articles empiriques } \\
\text { sont en augmentation. }\end{array}$ \\
\hline Meyer et al & \begin{tabular}{|c|} 
Utilisation \\
de la base \\
des données \\
Web of \\
Web of \\
Knowledge; \\
\end{tabular} & $\begin{array}{c}\text { de } 1990 \text { à } \\
2009\end{array}$ & $\begin{array}{l}\text { Utilisatio } \\
\quad \text { n de } \\
\text { l’analyse } \\
\text { structurel } \\
\quad \text { le }\end{array}$ & $\begin{array}{c}\text { le processus entrepreneurial } \\
\text { proposé par Shane et } \\
\text { Venkataraman dans la revue The } \\
\text { Academy of Management } \\
\text { Review en } 2000 \text { est le plus cité } \\
\text { avec } 1200 \text { citations. } \\
\end{array}$ \\
\hline $\begin{array}{l}\text { Frank Lasch et } \\
\text { Saïd Yami }\end{array}$ & $\begin{array}{l}253 \text { articles } \\
\text { et } \\
\text { communicat } \\
\text { ions de } 2 \\
\text { revues et } 2 \\
\text { conférences }\end{array}$ & $\begin{array}{c}\text { de } 1995 \text { à } \\
2005\end{array}$ & $\begin{array}{c}\text { Revue } \\
\text { de la } \\
\text { littérature }\end{array}$ & $\begin{array}{c}\text { Il y a une préférence de } \\
\text { méthodes qualitatives, } \\
\text { - le processus entrepreneurial est } \\
\text { privilégié comme thème de } \\
\text { recherche. } \\
\text { - la diffusion des connaissances } \\
\text { est encore insuffisante au niveau } \\
\text { international. }\end{array}$ \\
\hline $\begin{array}{c}\text { José C. Sánchez } \\
\text { Andrea Gutiérrez } \\
\text { (2011) }\end{array}$ & 471 articles & $\begin{array}{c}\text { de } 1977 \text { à } \\
2009\end{array}$ & $\begin{array}{c}\text { Revue } \\
\text { de la } \\
\text { littérature }\end{array}$ & $\begin{array}{c}\text { Il y a une préférence de } \\
\text { méthodes qualitatives, } \\
\text { - le processus entrepreneurial en } \\
\text { tant que thème de recherche est } \\
\text { privilégié, Cependant, } \\
\text { essentiellement en espagnole la } \\
\text { diffusion des connaissances } \\
\text { encore insuffisante au niveau } \\
\text { international ce qui limite sa } \\
\text { diffusion } \\
\end{array}$ \\
\hline
\end{tabular}


Cette revue de la littérature succincte laisse entendre qu'il y'a quelques similarités et beaucoup de divergences entre les recherches anglosaxonnes et les recherches dans les pays francophones et hispanophones.

En effet, d'une manière générale, la recherche en entrepreneuriat reste faible en termes de pourcentage par rapport aux travaux en management, et ce taux à tendance à évoluer depuis les deux dernières décennies. Par contre, au niveau des méthodes de recherches, les auteurs anglo-saxons ont une préférence pour les méthodes quantitatives, contrairement aux auteurs français.

\section{Méthodologie de recherche}

Pour dresser un état des lieux de la recherche en PME et entrepreneuriat au Maroc on a trouvé quelques difficultés, dues à l'absence de bases de données dédies aux revues nationales de gestion. De même que les revues publiées sont plutôt des revues généralistes orientés vers l'économie et le droit.

Donc, la rareté de revues spécialisées dans le domaine de la recherche, nous a poussé a sélectionner les trois principales revues en la matière qui sont : La Revue marocaine de contrôle de gestion (RMCG), la Revue Marocaine de Commerce de Gestion de l'École Nationale de Commerce et de Gestion de Tanger (RMCG-Tanger) et la Revue Marocaine de Recherches en Management et marketing (REMAREM).

De ce fait, on a réalisé une analyse bibliographique de ces revues, et qui se base sur l'analyse de 237 articles publiés entre 2005 et 2013

Nous présenterons dans cette section, l'échantillon étudié, ainsi que la codification utilisée pour faire notre recherche.

\section{L'échantillon étudié}

Nous présenterons dans un premier temps les trois revues objet de notre recherche, puis dans un deuxième temps la production des articles en gestion par revue et enfin la production scientifique Entrepreneuriat en dernier.

Tableau 3 - Répartition des articles en gestion par revue, numéros et effectif

\begin{tabular}{|c||c||c||c||c|}
\hline Revues & Années & $\begin{array}{c}\text { Nombre de } \\
\text { numéros }\end{array}$ & $\begin{array}{c}\text { Nombre } \\
\text { d'articles }\end{array}$ & $\begin{array}{c}\text { Pourcent } \\
\text { age }\end{array}$ \\
\hline \hline RMCG & $2009,2011,212$ & 4 & 57 & $24 \%$ \\
\hline \hline $\begin{array}{c}\text { RMCG } \\
\text { Tanger }\end{array}$ & $\begin{array}{c}2005,2006,2007,2008,2009, \\
2010,2012\end{array}$ & 7 & 69 & $29 \%$ \\
\hline \hline $\begin{array}{c}\text { REMARE } \\
\text { M }\end{array}$ & $2009,2010,2011,2012$ & 8 & 111 & $47 \%$ \\
\hline \hline & Total & 19 & 237 & $100 \%$ \\
\hline \hline
\end{tabular}


On constate que la revue REMAREM produit plus de $47 \%$ des articles en gestion, suivie de la revue RMCG de Tanger avec 29\%, la revue Marocaine de Contrôle de Gestion se place en dernière position avec 24\%. Cette différence peut être expliquée par la ligne éditoriale des deux premières revues qui sont généralistes, alors que la revue marocaine de contrôle de gestion est plus spécialisée.

Tableau 4 : Répartition annuelle des articles en PME et Entrepreneuriat

\begin{tabular}{|c|c|c|c|c|}
\hline Année & RMCG & RMCG Tanger & $\begin{array}{c}\text { REMARE } \\
M \\
\end{array}$ & Total \\
\hline 2005 & & 1 & & 1 \\
\hline 2006 & & 2 & & 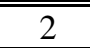 \\
\hline 2007 & & 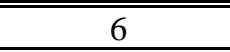 & & 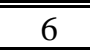 \\
\hline 2008 & & 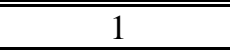 & & 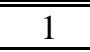 \\
\hline 2009 & & 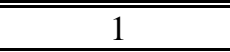 & 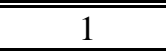 & 2 \\
\hline 2010 & & 3 & 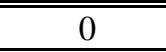 & 3 \\
\hline 2011 & 3 & 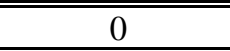 & 3 & 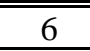 \\
\hline 2012 & 2 & 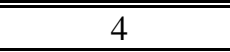 & 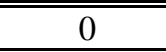 & 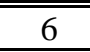 \\
\hline Total & 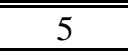 & 18 & 6 & 29 \\
\hline
\end{tabular}

De l'observation du tableau on constate que la production scientifique marocaine en Entrepreneuriat est encore faible, avec un taux de $12 \%$ de l'ensemble de la production marocaine en management de 2009 à 2012.

On remarque aussi, que la revue RMCG de Tanger a une orientation éditoriale vers le champ de l'entrepreneuriat avec une production de 18 articles soit $62 \%$ de la production totale en entrepreneuriat, suivi de la revue REMAREM avec 21\% et enfin la RMCG avec 17\%.

\section{Codification des éléments de l’analyse}

Pour analyser les articles en PME et entrepreneuriat, on s'est basé sur l'étude des descripteurs (2.2.1), la typologie méthodologique (2.2.2) et enfin le comptage des citations.

\section{Codification des concepts de PME et entrepreneuriat}

Pour décrire le concept de PME et d'entrepreneuriat plusieurs descripteurs sont généralement utilisés, nous inspirant de la recherche de Kizaba (2006) qui a utilisé la grille suivante :

- Entrepreneur,

- $\quad$ Entreprenariat,

- $\quad$ Entrepreneuriat,

- Innovation,

- $\quad$ Organisation entrepreneuriale, 
- $\quad$ Management entrepreneurial,

- $\quad$ Entreprise entrepreneurial

- $\quad$ Esprit d'entreprise (d'entreprendre),

- $\quad$ Création d'entreprise (d'activité),

- $\quad$ PME, PMI, PE, TPE

\section{Codification des méthodologies utilisées dans les articles}

On a mobilisé la classification employée par Needles, Jr (1997) pour étudier les différentes méthodes de recherches utilisées par les auteurs, elle se décline en six classes :

- $\quad$ Méthodologie quantitative,

- $\quad$ Méthodologie qualitative,

- $\quad$ La modélisation,

- $\quad$ La méthodologie historique,

- $\quad$ La revue de la littérature,

- $\quad$ Et la discussion.

\section{Codification des contributions}

Lorsqu'un article est co-écrit par plusieurs auteurs, la contribution de chaque auteur est mesurée par une fraction.

\section{Résultats et discussion}

Nous présentons, tout d'abord les résultats relatifs à la nationalité des auteurs et à leur influence (4.1), puis ceux qui concernent ses principales thématiques étudiées (4.2) et enfin ceux qui sont relatifs aux méthodologies mobilisées (4.3).

Nationalité et productivité des principaux auteurs et instituts de formation en entrepreneuriat

On remarque que la majorité des articles est publiée en langue française, et que la ajorité des conseils éditoriaux sont constitués par des grands noms de la recherche française en gestion, ce qui dénote de l'influence de l'école française sur la recherche marocaine en management en général et en entrepreneuriat en particulier.

\section{Nationalité des principaux auteurs en entreprenariat}

On constate aussi que $60 \%$ des auteurs publiant dans les trois revues sont de nationalité marocaine (Tableau 5) et 19\% sont des auteurs français ou co-auteurs franco-marocains, $12 \%$ sont des maghrébins et le reste $9 \%$ d'autres nationalités. 
Tableau 5 : Répartition des chercheurs par nationalité

\begin{tabular}{|c||c||c|}
\hline Nationalité & Effectif des auteurs & Pourcentage \\
\hline \hline Marocaine & 26 & $60 \%$ \\
\hline \hline Française & 8 & $19 \%$ \\
\hline \hline Tunisie & 3 & $7 \%$ \\
\hline \hline Algérienne & 2 & $5 \%$ \\
\hline \hline Italie & 2 & $5 \%$ \\
\hline \hline Turque & 1 & $2 \%$ \\
\hline \hline Iranienne & 1 & $2 \%$ \\
\hline \hline Total & 43 & $100 \%$ \\
\hline
\end{tabular}

\section{Productivité des principaux auteurs et instituts de formation}

De l'observation du tableau 6, on constate que'au niveau institutionnel, l’ENCG de Tanger produit à elle seule, plus de 50\% de la recherche en Entrepreneuriat, suivi par les facultés de droit (Meknès, Rabat et Fès) par 30\%, et enfin le réseau français des Instituts d’administration des entreprises (Toulon et Nice) par 20\%.

Tableau 6 : Productivité des institutions de recherche en Entrepreneuriat

\begin{tabular}{|c||c||c|}
\hline Institution & Nationalité & Classement \\
\hline \hline ENCG Tanger & Marocaine & $52 \%$ \\
\hline \hline IAE Toulon-Var & Française & $16 \%$ \\
\hline \hline IAE de Nice & Française & $8 \%$ \\
\hline \hline Fsjes, M5-Rabat & Marocaine & $8 \%$ \\
\hline \hline Fsjes, Fès & Marocaine & $8 \%$ \\
\hline \hline Fsjes,Meknes & Marocaine & $8 \%$ \\
\hline \multicolumn{2}{|c|}{ Total } & $100 \%$ \\
\hline
\end{tabular}

\section{Principales thématiques étudiées}

En adéquation avec ce qui se fait au niveau de la recherche francophone en Entrepreneuriat, le thème de la PME arrive en tête avec 19 articles (66\%), suivi de l'entrepreneuriat avec 10 articles (34\%).

Tableau 7 : Répartition des différents thèmes de recherche en Entrepreneuriat

\begin{tabular}{|c||c||c||c|}
\hline Revues/ Thèmes & PME & Entrepreneuriat & Total \\
\hline \hline RMCG & 5 & 0 & 5 \\
\hline \hline RMCG Tanger & 9 & 9 & 18 \\
\hline \hline Remarem & 5 & 1 & 6 \\
\hline \hline Poids & 19 & 10 & $100 \%$ \\
\hline \hline Pourcentage & $66 \%$ & $34 \%$ & \\
\hline
\end{tabular}




\section{Les différentes méthodologies mobilisées dans la recherche en entrepreneuriat}

La revue de la littérature arrive en tête des méthodes de recherches utilisées par les chercheurs avec 19 articles (66\%), suivi par les méthodes qualitatives avec 8 articles. La méthodologie quantitative arrive en dernière position avec 2 articles (7\%).

En adéquation avec F Lasch et S Yami (2008), qu’il y’a aussi une préférence des chercheurs marocains pour les méthodologies qualitatives due à l'effet « French Touch ».

Tableau 8 : Les méthodes de recherches utilisées

\begin{tabular}{|c||c||c||c||c|}
\hline Méthodologie & Quantitative & Qualitative & Revue de la littérature & Total \\
\hline \hline RMCG & 0 & 1 & 4 & 5 \\
\hline \hline RMCG Tanger & 1 & 6 & 11 & 18 \\
\hline \hline REMAREM & 1 & 1 & 4 & 6 \\
\hline \hline TOTAL & 2 & 8 & 19 & 29 \\
\hline \hline Pourcentage & $7 \%$ & $28 \%$ & $66 \%$ & $100 \%$ \\
\hline
\end{tabular}

\section{Conclusion}

L’objectif de cet article était la présentation des principaux résultats relatifs à une étude bibliographique sur la recherche marocaine en Entrepreneuriat.

Cette étude est la première au Maroc à avoir essayé d’évaluer la recherche scientifique en Entrepreneuriat et PME.

Les résultats suivants doivent être soulignés:

En adéquation avec la littérature citée, la production scientifique marocaine en Entrepreneuriat est encore faible, avec une moyenne de 3.6 articles par an, sur la période de 2009 à 2012,

- De même les revues spécialement dédiées a ce domaine sont rares, en effet, la revue Marocaine de commerce et de gestion de Tanger est la principale revue orientée vers la PME et entrepreneuriat,

De même on observe que l'influence de l'école française en entrepreneuriat est très importante. Les sujets et les méthodes issus de ce pays dominent encore la recherche marocaine.

A la question principale de notre recherche: «la PME et l'entrepreneuriat font t'ils l'objet de recherche au Maroc? ». Notre réponse est mitigée :

- Oui, il y’a une certaine recherche dans ce domaine (29 articles sur une période de neuf ans), mais, le nombre de revues dédiées à ce champs de recherche, ainsi que la productivité est faible. Toute fois, La tendance est à la hausse depuis l'année 2011.

Les résultats de cette recherche doivent être pris avec précaution au vu des limites de notre travail. 
En effet, vue l'absence de bases de données de revues marocaines, on s'est limité aux revues publiées entre 2005 et 2013, et au niveau méthodologique, l'approche adoptée est une approche bibliographique, l'analyse bibliométrique à travers l'analyse des citation est une perspective prometteuse pour une recherche future.

\section{References :}

Ahsina, K (2012). Dix ans de recherche en Comptabilité, Contrôle et Audit au Maroc: une approche bibliographique, Revue du chercheur, $\mathrm{N}^{\circ} 11$.

Lowell W. Busenitz, and al (2003). "Entrepreneurship Research in Emergence: Past Trends and Future Directions” Journal of Management.

M. Meyer, D. Libaers, B. Thijs, K. Grant,W. Glänzel, K. Debackere”Origin and Emergence of Entrepreneurship as a Research Field"

Fayolle A. (2000). "Dynamisme entrepreneurial et croissance économique : une comparaison France-USA”, Histoire d'entreprendre - les réalités de l'entrepreneuriat, Verstraete T. (sous la direction de), Paris :EMS, Management et Société, p.33-48

Frank Lasch et Saïd Yami (2008). "The Nature and Focus of Entrepreneurship Research in France over the Last Decade: A French Touch?” Entrepreneurship Theory and Practice, Vol 32 Issue 2.

José C. Sánchez and Andrea Gutiérrez (2011). Entrepreneurship research in Spain: Developments and distinctiveness, Psicothema 2011. Vol. 23, $\mathrm{n}^{\circ} 3$, pp. 458-463

Taouab, O (2014). The Determinants of Entrepreneurial Act in the Moroccan Textile Sector: A Logistic Regression Approach. Business and Management Horizons, Vol 2, No 1 (2014) 